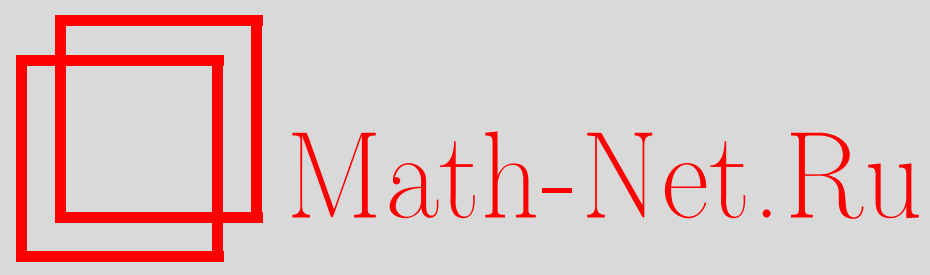

В. А. Колмыков, Преобразование Кокстера и квазиколчаны, Матем. заметки, 2002, том 72, выпуск 3, 472-473

DOI: https://doi.org/10.4213/mzm667

Использование Общероссийского математического портала Math-Net.Ru подразумевает, что вы прочитали и согласны с пользовательским соглашением http://www. mathnet.ru/rus/agreement

Параметры загрузки:

IP: 54.198 .55 .26

26 апреля 2023 г., 12:56:49

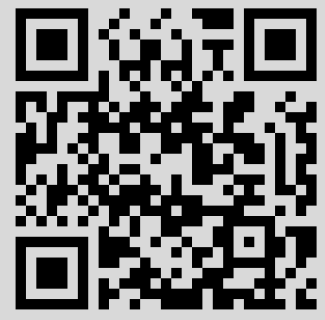




\section{ПРЕОБРАЗОВАНИЕ КОКСТЕРА И КВАЗИКОЛЧАНЫ}

\section{В. А. Колмыков}

Пусть $A$ - комплексная $(l \times l)$-матрица ${ }^{1}$. Если $U=U(i, j)$ - некоторое условие, наложенное на $i$ и $j$, то через $A_{(U)}$ обозначим матрицу, получающуюся из $A$, если в ней оставить все элементы, индексы которых удовлетворяют условию $U$, а остальные заменить нулями. Например, если $k-$ фиксированное число, то $A_{(j=k)}$ получается из $A$ занулением всех элементов, кроме тех, которые стоят в $k$-м столбце. Положим $R_{k}=R_{k}(A)=I-A_{(j=k)}$. Обозначим

$$
C(A)=R_{1}(A) R_{2}(A) \cdots R_{l}(A) .
$$

Преобразованием Кокстера, связанным с матрицей $A$, называется преобразование пространства строк $x \mapsto x C(A)$.

С матрицей $A$ можно связать граф $\Gamma_{A}$ следуюшим образом. Множество его вершин $I=\{1, \ldots, l\}$, множество ребер $S$ состоит из тех двухэлементных подмножеств $\{i, j\}$ множества $I$, для которых $a_{i j} \neq 0$ либо $a_{j i} \neq 0$.

Теорема [1, гл. $5, \S 6$, упр. 3]. Пусть $\Gamma_{A}-л е с$ u $a_{i i}=2$ для любы $x^{2} i$. Тогда

$$
\left|\lambda^{2} I-C(A)\right|=\lambda^{l}\left|\lambda I+\lambda^{-1} I+A_{(i \neq j)}\right| .
$$

В настоящей заметке мы ослабляем первое ограничение и снимаем второе.

Ориентированным графом назовем пару $(I, E)$, где $I$-конечное множество (вершин), $E \subseteq I^{2}$. Элемент $(i, j) \in E$ называется стрелкой с началом $i$ и концом $j$. Контуром длины $k \in \mathbb{Z} / k \mathbb{Z}$ в ориентированном графе называется последовательность $\left\{e_{1}, \ldots, e_{k}\right\}$ различных стрелок таких, что конец $e_{i}$ - это начало $e_{i+1}$ и ни одна вершина не является концом двух стрелок из этой последовательности. Колчан - это ориентированный граф̆, в котором нет контуров. Квазиколчаном назовем ориентированный граф, в котором нет контуров длины $\geqslant 3$.

С матрицей $A$ свяжем ориентированньй граф $O_{A}$ следуюшим образом: $I=\{1, \ldots, l\},(i, j) \in E$, тогда и только тогда, когда $a_{i j} \neq 0$.

ПримеР. Если $\Gamma_{A}-$ лес, то $O_{A}-$ квазиколчан.

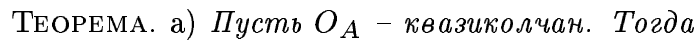

$$
\left|\lambda^{2} I-C(A)\right|=\lambda^{l}\left|\lambda I+\lambda^{-1}\left(A_{(i=j)}-I\right)+A_{(i \neq j)}\right| .
$$

б) Заменим в матрице $A$ нулями все әлементы $a_{i j}$ такие, что $a_{j i}=0$, и полученную матричу обозначим $\widetilde{A}$. Тогда $\Gamma_{\tilde{A}}-$ лес $и|\lambda I-C(A)|=|\lambda I-C(\widetilde{A})|$.

ДокАЗАтЕльство. а) Модифицируем рассуждения из [1, гл. 5, §6, упр. 3]. Определения вспомогательных конструкций процитированы оттуда же.

1) Сначала не будем накладывать ограничений на матрицу $A$. Для $\alpha=\{i, j\} \in S$ обозначим через $\sigma_{\alpha}$ транспозицию $i$ и $j$ (рассматриваемую как элемент симметрической группы $S_{l}$ ) и положим $a_{\alpha}=-a_{i j} a_{j i}$.

Пусть $\mathscr{X}$ - множество подмножеств $S$, состоящих из ребер с различными концами. Обозначим через $C(X), X \in \mathscr{X}$, множество тех $i \in I$, которые не являются концами ребер из $X$, и положим

$$
\sigma_{X}=\prod_{\alpha \in X} \sigma \alpha, \quad a_{X}=\prod_{\alpha \in X} a_{\alpha} .
$$

\footnotetext{
${ }^{1}$ Все результаты заметки верны и в более общем (принятом Н. Бурбаки) случае, следует только заменить $\mathbf{C}$ на коммутативное кольо $K$, а пространство строк - на $K$-модуль строк.

${ }^{2}$ Во французском и в русском изданиях [1] опечатка. Написано $a_{i i}=1$, следует читать $a_{i i}=2$.
} 
В $[1$, гл. $5, \S 6$, упр. 3$]$ было доказано, что

$$
|\lambda I-C(A)|=\left|A_{(i>j)}+\left((\lambda-1) I+A_{(i=j)}\right)+\lambda A_{(i<j)}\right| .
$$

Рассмотрим определитель, стоящий в правой части. Пусть $\sigma \in S_{l}$ и пусть $d_{\sigma}-$ член, соответствующий $\sigma$ в разложении этого определителя. Если $\sigma$ имеет вид $\sigma_{X}$ с $X \in \mathscr{X}$, то

$$
d_{\sigma}=a_{X} \lambda^{\operatorname{Card}(X)} \prod_{i \in C(X)}\left(\lambda+a_{i i}-1\right) .
$$

Положим

$$
f_{A}(\lambda)=\left|\lambda I+\lambda^{-1}\left(A_{(i=j)}-I\right)+A_{(i \neq j)}\right| .
$$

Рассмотрим определитель, стоящий в правой части. Пусть $\sigma \in S_{l}$ и пусть $D_{\sigma}-$ член, соответствующий $\sigma$ в разложении этого определителя. Если $\sigma$ имеет вид $\sigma_{X}$ с $X \in \mathscr{X}$, то

$$
D_{\sigma}=a_{X} \prod_{i \in C(X)}\left(\lambda+\lambda^{-1}\left(a_{i i}-1\right)\right) .
$$

2) Предположим теперь, что $O_{A}-$ квазиколчан. Если $\sigma \in S_{l}$ не имеет вида $\sigma_{X}$ с $X \in \mathscr{X}$, то $d_{\sigma}=D_{\sigma}=0$ (действительно, если в каноническом разложении $\sigma$ в произведение независимых цик лов имеется цикл длины $\geqslant 3$, то, двигаясь по вершинам квазиколчана $O_{A}$ вслед за этим циклом, мы сойдем с $O_{A}$, т.е. соответствующий элемент $a_{i j}$ равен нулю, но $a_{i j}$ - множитель из $d_{\sigma}$ и $\left.D_{\sigma}\right)$. Отсюда

$$
\begin{gathered}
|\lambda I-C(A)|=\sum_{X \in \mathscr{X}} a_{X} \lambda^{\operatorname{Card}(X)} \prod_{i \in C(X)}\left(\lambda+a_{i i}-1\right), \\
f_{A}(\lambda)=\sum_{X \in \mathscr{X}} a_{X} \prod_{i \in C(X)}\left(\lambda+\lambda^{-1}\left(a_{i i}-1\right)\right)
\end{gathered}
$$

поэтому

$$
\left|\lambda^{2} I-C(A)\right|=\lambda^{l} \sum_{X \in \mathscr{X}} a_{X} \prod_{i \in C(X)}\left(\lambda+\lambda^{-1}\left(a_{i i}-1\right)\right),
$$

т.е. $\left|\lambda^{2} I-C(A)\right|=\lambda^{l} f_{A}(\lambda)$.

б) Если бы $\Gamma_{\widetilde{A}}$ не был лесом, то в нем нашелся бы простой цикл длины $\geqslant 3$, каждому его ребру соответствуют две противоположные стрелки в квазиколчане $O_{\tilde{A}}$, т.е. в этом квазиколчане был бы контур длины $\geqslant 3$; противоречие. Если $a_{j i}=0$, то стрелка $(i, j)$ не принадлежит никакому контуру квазиколчана $O_{A}$ (в контур длины 2 она не входит, так как стрелки $(j, i)$ нет, а контуров длины $\geqslant 3$ в квазиколчане нет), поэтому $a_{i j}$ не входит множителем ни в какое ненулевое слагаемое $d_{\sigma}$.

\section{СПИСОК ЦИТИРОВАННОЙ ЛИТЕРАТУРЫ}

1. Бурбаки Н. Группы и алгебры Ли. Группы Кокстера и системы Титса. Группы, порожденные отражениями. Системы корней. М.: Мир, 1972. 